\title{
Infinite Matrix Products and the Representation of the Matrix Gamma Function
}

\author{
J.-C. Cortés, ${ }^{1}$ L. Jódar, ${ }^{1}$ Francisco J. Solís, ${ }^{2}$ and Roberto Ku-Carrillo ${ }^{3}$ \\ ${ }^{1}$ Instituto Universitario de Matemática Multidisciplinar, Universitat Politècnica de València, Camino de Vera $s / n$, \\ 46022 Valencia, Spain \\ ${ }^{2}$ CIMAT, 36000 Guanajuato, GTO, Mexico \\ ${ }^{3}$ Universidad Autónoma de Aguascalientes, 20131 Aguascalientes, AGS, Mexico
}

Correspondence should be addressed to J.-C. Cortés; jccortes@imm.upv.es

Received 24 October 2015; Accepted 25 November 2015

Academic Editor: Allan Peterson

Copyright (c) 2015 J.-C. Cortés et al. This is an open access article distributed under the Creative Commons Attribution License, which permits unrestricted use, distribution, and reproduction in any medium, provided the original work is properly cited.

We introduce infinite matrix products including some of their main properties and convergence results. We apply them in order to extend to the matrix scenario the definition of the scalar gamma function given by an infinite product due to Weierstrass. A limit representation of the matrix gamma function is also provided.

\section{Introduction}

Scalar special functions play a significant role in applied mathematics, Physics, engineering, economics, and industry. From a mathematical point of view, many special functions have been introduced for describing the solutions of certain second-order differential equations [1]. They also appear in connection with orthogonal polynomials and their fruitful applications in Physics, particularly in the theory of Lie groups and Lie algebras $[2,3]$. During the last few decades numerous contributions have focused on generalizing the well-developed scalar theory on special functions to their matrix analogous counterpart and this extension continues being an active area under research [4-6].

Among special functions, the gamma function plays a distinguished role due to its ubiquity in mathematics. The gamma function appears in areas as seemingly different as Number Theory (it generalizes the concept of factorial for complex numbers), probability (takes part in the definition of relevant probability density functions like the gamma distribution and some of its recent generalizations [7]), and differential equations (appears in solving significant continuous models like the Bessel equation) and recently it has been used as a cornerstone to develop the fractional calculus since it permits expressing the fractional derivative of certain functions including the potential-type functions.
Although the scalar gamma function possesses several representations, its integral form is the most widely used one. It has done that matrix generalizations of the gamma function had mainly focused on integral expressions. In this sense, some relevant contributions focussing on the integral generalization to the matrix scenario of the gamma function and its properties including its relationship with other special functions and statistical counterpart have been made recently $[8,9]$. Apart from the aforementioned integral representation of the gamma function, it can also be expressed by an infinite product. Such representation, due to Weierstrass, plays a prominent role in dealing with other special functions such as the digamma or more generally polygamma functions closely related to numerous problems that appear in Number Theory [10]. This fact motivates the extension to the matrix framework of the Weierstrass definition to the gamma function which, in addition, entails the generalization of some required results related to infinite matrix products. Some interesting results regarding matrix infinite products are available (see, e.g., [11-13]); to the best of our knowledge, none of them includes the ones that will be presented in Section 3.

The aim of this paper is to introduce infinite matrix products and their main properties and convergence results, with our main focus to extend the gamma function defined by Weierstrass as an infinite product to the matrix scenario. 
Taking advantage of such definition we also provide a limit representation of the matrix gamma function. With these benefits, it is hoped that our approach provides an alternative method to the existing ones that may open up new avenues to the use of the matrix gamma function in practical applications.

The paper is organized as follows. Section 2 summarizes the main results and definitions that will be used throughout the paper. Section 3 introduces infinite matrix products and some relevant results regarding their convergence. The matrix extension of the scalar definition of the gamma function due to Weierstrass through an infinite product is presented in Section 4. A limit representation of the matrix gamma function is also included in this section. Conclusions are drawn in Section 5.

\section{Preliminaries}

As we mentioned in the previous section, our goal is twofold: first, we pursue extending from the scalar framework the main results for matrix infinite products and, second, applying them in order to generalize the classical definition of the gamma function due to Weierstrass in the matrix case. The scalar gamma function given by Weierstrass is defined as

$$
\frac{1}{\Gamma(z)}=z e^{\gamma z} \prod_{n=1}^{\infty}\left[\left(1+\frac{z}{n}\right) e^{-z / n}\right], \quad z \in \mathbb{C},
$$

where $\gamma$ is the Euler-Mascheroni constant given by

$$
\gamma=\lim _{n \rightarrow \infty}\left(H_{n}-\ln n\right) \approx 0.5772, \quad H_{n}=\sum_{k=1}^{n} \frac{1}{k} .
$$

For the sake of clarity in the presentation, in the following we summarize the main definitions and results that will be used throughout the paper (see $[14,15]$ for further details). The set of all the square matrices of size $r$ whose entries are complex numbers will be denoted by $\mathbb{C}^{r \times r}$.

Definition 1. Given $C \in \mathbb{C}^{r \times r}$, the spectrum of $C$ is the set of all eigenvalues of $C$ and it is denoted by $\sigma(C)$. The number $r(C)$ given by $r(C)=\max \{|\lambda|: \lambda \in \sigma(C)\}$ is called the spectral radius of $C$.

Definition 2. The associated 2-norm of a matrix $C \in$ $\mathbb{C}^{r \times r}$, denoted by $\|C\|$, is defined by the following: $\|C\|=$ $\sup _{x \neq 0}\left(\|C x\|_{2} /\|x\|_{2}\right)$, where $x \in \mathbb{C}^{r}$ and $\|x\|_{2}$ is the usual Euclidean norm of $x$.

The following relationship between the spectral radius and the 2-norm is well known: $r(C) \leq\|C\|$.

The following result will be used later. We omit its proof because it is a direct consequence of the definition of the spectrum of a matrix.

Proposition 3. Let $A \in \mathbb{C}^{r \times r}$ and $I \in \mathbb{C}^{r \times r}$ be the identity matrix; then $I+A$ is invertible in $\mathbb{C}^{r \times r}$ if and only if $-1 \notin \sigma(A)$.

The following result permits extending the concept of Taylor series to a function of matrices.
Proposition 4 (Th. 11.2.3 of [15, pages 549-550]). If $f(z)$ has a power series representation on an open disk which contains the spectrum of $A$, that is,

$$
\begin{aligned}
& f(z)=\sum_{n=0}^{\infty} c_{n} z^{n}, \\
& \quad z \in D_{\delta}=\{z \in \mathbb{C}:|z|<\delta\}: \sigma(A) \subset D_{\delta},
\end{aligned}
$$

then

$$
f(A)=\sum_{n=0}^{\infty} c_{n} A^{n}
$$

As a direct consequence of the previous results one gets the following.

Proposition 5. Let $A \in \mathbb{C}^{r \times r}$ such that $\|A\|<1$. Then

$$
\ln (I+\mathrm{A})=\sum_{n=1}^{\infty} \frac{(-1)^{n-1}}{n} A^{n}
$$

where $\ln (\cdot)$ denotes the logarithmic function in the natural base e.

Throughout this paper the exponential of a square matrix is defined as usual [15]:

$$
e^{A}=\sum_{n=0}^{\infty} \frac{A^{n}}{n !}, \quad A \in \mathbb{C}^{r \times r} .
$$

From this definition one gets $e^{O}=I$, where $O$ and $I$ denote the null and identity matrices in $\mathbb{C}^{r \times r}$, respectively. The following algebraic identity that will be used later can be proven easily from representation (6).

Proposition 6. Let $A, B \in \mathbb{C}^{r \times r}$ be such that $A$ and $B$ commute. Then

$$
e^{A+B}=e^{A} e^{B}
$$

\section{Infinite Matrix Products}

In this section we first extend to the matrix framework the definition of convergence to an infinite product of square matrices. We then establish some results related to the convergence of infinite matrix products. These results generalize their scalar counterpart including a characterization of the absolute convergence of infinite matrix products in terms of the associated logarithmic matrix series which is particularly useful in practice.

Definition 7. Let $\left\{A_{n}\right\}_{n=1}^{\infty}$ be a sequence of matrices in $\mathbb{C}^{r \times r}$ and consider the finite matrix product:

$$
P_{n}=\prod_{k=1}^{n}\left(I+A_{k}\right)=\left(I+A_{1}\right)\left(I+A_{2}\right) \cdots\left(I+A_{n}\right),
$$

where $I \in \mathbb{C}^{r \times r}$ denotes the identity matrix. If the limit $\lim _{n \rightarrow \infty} P_{n}$ exists and its value is an invertible matrix denoted 
by $P \in \mathbb{C}^{r \times r}$, we say that the infinite matrix product $\prod_{k=1}^{n}(I+$ $A_{k}$ ) exists and the matrix $P$ is its value. Then, we write

$$
\prod_{n=1}^{\infty}\left(I+A_{n}\right)=P .
$$

$P_{n}$ in (8) is referred to as the $n$th partial product, and $I+A_{n}$ in (9) is called the general term of the infinite product.

Remark 8. Notice that taking determinants in (9) since $P$ is invertible by the continuity of the determinant function there exists $n_{0}$ such that $\operatorname{det}\left(P_{n}\right) \neq 0 \forall n \geq n_{0}$. Thus in (9) there are at most a finite number of singular factors. In such case if the rest of the product converges to an invertible matrix we say that the original product converges to the null matrix. This motivates the fact that in the following we will not consider infinite products with an infinite number of singular factors. In the case that only a finite number of factors are singular, we will discard them from the matrix product and we will deal with the rest of the infinite matrix product. Then, in accordance with Proposition 3 and without loss of generality, this is equivalent to assume in the context of Definition 7 that $-1 \notin \sigma\left(A_{n}\right), \forall n \geq 1$.

Now we will establish the following necessary condition for the convergence of an infinite matrix product which is analogous to the corresponding one for infinite series.

Theorem 9. Let $\left\{A_{n}\right\}_{n=1}^{\infty}$ be a sequence of matrices in $\mathbb{C}^{r \times r}$. If the infinite matrix product $\prod_{n=1}^{\infty}\left(I+A_{n}\right)$ exists, then

$$
\lim _{n \rightarrow \infty} A_{n}=0 \in \mathbb{C}^{r \times r} .
$$

Proof. First, we deal with the case that $-1 \notin \sigma\left(A_{n}\right), \forall n \geq 1$; that is, there are no singular terms among the factors of the infinite product. By assumption $\prod_{n=1}^{\infty}\left(I+A_{n}\right)$ exists; hence we can define $P$ as

$$
P=\prod_{n=1}^{\infty}\left(I+A_{n}\right)=\lim _{n \rightarrow \infty} \prod_{k=1}^{n}\left(I+A_{k}\right) .
$$

Thus, taking into account the continuity of the inverse function $x^{-1}$ as well as the inverse of a finite product of invertible matrices, one gets

$$
\begin{aligned}
I & =P^{-1} P=\left[\lim _{n \rightarrow \infty} \prod_{k=1}^{n-1}\left(I+A_{k}\right)\right]^{-1} \\
& \cdot\left[\lim _{n \rightarrow \infty} \prod_{k=1}^{n}\left(I+A_{k}\right)\right]=\lim _{n \rightarrow \infty}\left[\prod_{k=1}^{n-1}\left(I+A_{k}\right)\right]^{-1} \\
& \cdot \lim _{n \rightarrow \infty}\left[\prod_{k=1}^{n}\left(I+A_{k}\right)\right] \\
& =\lim _{n \rightarrow \infty}\left\{\left[\prod_{k=1}^{n-1}\left(I+A_{k}\right)\right]^{-1}\left[\prod_{k=1}^{n}\left(I+A_{k}\right)\right]\right\} \\
& =\lim _{n \rightarrow \infty}\left\{\left(I+A_{n-1}\right)^{-1}\left(I+A_{n-2}\right)^{-1} \cdots\left(I+A_{1}\right)^{-1}\right\}
\end{aligned}
$$

$$
\begin{aligned}
& \cdot\left\{\left(I+A_{1}\right) \cdots\left(I+A_{n-1}\right)\left(I+A_{n}\right)\right\} \\
& =\lim _{n \rightarrow \infty}\left(I+A_{n}\right)=I+\lim _{n \rightarrow \infty} A_{n} .
\end{aligned}
$$

So we have shown that $I=I+\lim _{n \rightarrow \infty} A_{n}$; therefore $\lim _{n \rightarrow \infty} A_{n}=0 \in \mathbb{C}^{r \times r}$. If there are singular factors in the matrix infinite product, we discard them and repeat the previous argument.

3.1. Associated Matrix Logarithm Series. To every infinite matrix product we can associate an infinite matrix series whose terms are logarithms. In the following result, we prove that both the infinite product and series have the same character. As a consequence, the character of an infinite matrix product can be studied by means of an infinite matrix series for which a considerable number of well-established tests are available.

Theorem 10. Let $\left\{A_{n}\right\}_{n=1}^{\infty}$ be a sequence of matrices in $\mathbb{C}^{r \times r}$ such that $A_{n} A_{m}=A_{m} A_{n}$ for every $n, m \geq 1$. Then

$$
\begin{aligned}
& \prod_{n=1}^{\infty}\left(I+A_{n}\right), \\
& \sum_{n=1}^{\infty} \ln \left(I+A_{n}\right)
\end{aligned}
$$

have the same character; that is, both converge or diverge.

Proof. Since we are assuming that $-1 \notin \sigma\left(A_{n}\right), \forall n \geq 1$ (see Remark 8), every matrix term of the form $I+A_{n}$ is invertible and its logarithm exists [16]. If the infinite matrix product has (a finite number of) singular factors, we remove them.

Let us denote by $S_{n}$ and $P_{n}$ the partial sum and the partial product of (13), respectively:

$$
\begin{aligned}
& S_{n}=\sum_{k=1}^{n} \ln \left(I+A_{k}\right), \\
& P_{n}=\prod_{k=1}^{n}\left(I+A_{k}\right) .
\end{aligned}
$$

Using Proposition 6 (since $I+A_{n}$ and $I+A_{n}$ commute because $A_{n}$ and $A_{m}$ also do by hypothesis),

$$
\begin{aligned}
e^{S_{n}} & =e^{\sum_{k=1}^{n} \ln \left(I+A_{k}\right)}=e^{\ln \left(I+A_{1}\right)} e^{\ln \left(I+A_{2}\right)} \cdots e^{\ln \left(I+A_{n}\right)} \\
& =\left(I+A_{1}\right)\left(I+A_{2}\right) \cdots\left(I+A_{n}\right)=\prod_{k=1}^{n}\left(I+A_{k}\right) \\
& =P_{n} .
\end{aligned}
$$

Then $e^{S_{n}}=P_{n}$. Taking limits when $n \rightarrow \infty$ one gets

$$
\lim _{n \rightarrow \infty} e^{S_{n}}=\lim _{n \rightarrow \infty} P_{n} \text {. }
$$

Since

$$
\lim _{n \rightarrow \infty} e^{S_{n}}=e^{\lim _{n \rightarrow \infty} S_{n}}
$$


we conclude

$$
e^{\lim _{n \rightarrow \infty} S_{n}}=\lim _{n \rightarrow \infty} P_{n} .
$$

Therefore $P_{n}$ has a limit if and only if $S_{n}$ has a limit. Thus $\prod_{n=1}^{\infty}\left(I+A_{n}\right)$ and $\sum_{n=1}^{\infty} \ln \left(I+A_{n}\right)$ have the same character.

Remark 11. Notice that from (18) we conclude that $P_{n}$ approaches an invertible matrix since the exponential matrix is invertible, as it was required in the definition of convergence of an infinite product where all the singular factors are assumed to be previously removed.

3.2. Absolute Convergence. On account of Theorem 10, we can define the absolute convergence of an infinite matrix product in terms of the associated series of matrix logarithms. For it, we remember again that we assume that the infinite matrix product $\prod_{n=1}^{\infty}\left(I+A_{n}\right)$ has had its singular factors, if any, deleted.

Definition 12. One says that the infinite matrix product $\prod_{n=1}^{\infty}\left(I+A_{n}\right)$ is absolutely convergent if and only if the infinite matrix series $\sum_{n=1}^{\infty} \ln \left(I+A_{n}\right)$ is absolutely convergent.

The following result shows that convergence absolute of an infinite matrix product can be characterized in terms of the absolute convergence of an infinite matrix series.

Theorem 13. Let $\left\{A_{n}\right\}_{n=1}^{\infty}$ be a sequence of matrices in $\mathbb{C}^{r \times r}$ such that $A_{n} A_{m}=A_{m} A_{n}$ for every $n, m \geq 1$. Then $\prod_{n=1}^{\infty}(I+$ $A_{n}$ ) absolutely converges if and only if $\sum_{n=1}^{\infty} A_{n}$ absolutely converges.

Proof. Taking into account Definition 12, it is enough to prove that $\sum_{n=1}^{\infty} \ln \left(I+A_{n}\right)$ is absolutely convergent if and only if $\sum_{n=1}^{\infty} A_{n}$ is absolutely convergent; that is,

$$
\begin{gathered}
\sum_{n=1}^{\infty}\left\|\ln \left(I+A_{n}\right)\right\|<\infty \Longleftrightarrow \\
\sum_{n=1}^{\infty}\left\|A_{n}\right\|<\infty .
\end{gathered}
$$

Let us prove both implications simultaneously. Since $\prod_{n=1}^{\infty}(I+$ $A_{n}$ ) and $\sum_{n=1}^{\infty}\left\|A_{n}\right\|$ are convergent, then using Theorem 9 in the product case and the necessary convergence condition for matrix series, one gets

$$
\lim _{n \rightarrow \infty} A_{n}=0 \in \mathbb{C}^{r \times r} .
$$

Then, there exists $n_{0} \in \mathbb{N}$, such that $\left\|A_{n}\right\|<1 / 2$ for all $n>n_{0}$. As a consequence, by Proposition 5 , the following logarithmic matrix function is well defined:

$$
\begin{aligned}
& \ln \left(I+A_{n}\right)=\sum_{k=1}^{\infty} \frac{(-1)^{k-1}}{k}\left(A_{n}\right)^{k}, \\
& \forall n>n_{0}:\left\|A_{n}\right\|<\frac{1}{2} .
\end{aligned}
$$

Then, for each $n>n_{0}$ such that $\left\|A_{n}\right\|<1 / 2$, applying the triangle inequality and submultiplicativity of the matrix norm, one gets

$$
\begin{aligned}
& \left|\left\|\ln \left(I+A_{n}\right)\right\|\left\|A_{n}\right\|^{-1}-1\right| \\
& \quad=|| \sum_{k=1}^{\infty} \frac{(-1)^{k-1}}{k}\left(A_{n}\right)^{k}\|\| A_{n} \|^{-1}-1 \mid \\
& \quad \leq\left|\left(\sum_{k=1}^{\infty} \frac{\left\|A_{n}\right\|^{k}}{k}\right)\left\|A_{n}\right\|^{-1}-1\right|=\left|\left(\sum_{k=1}^{\infty} \frac{\left\|A_{n}\right\|^{k-1}}{k}\right)-1\right| \\
& \quad=\left|\sum_{k=2}^{\infty} \frac{\left\|A_{n}\right\|^{k-1}}{k}\right|=\left|\sum_{k=1}^{\infty} \frac{\left\|A_{n}\right\|^{k}}{k+1}\right| \\
& \quad \leq \sum_{k=1}^{\infty} \frac{1}{k+1}\left(\frac{1}{2}\right)^{k}<\sum_{k=1}^{\infty} \frac{1}{2}\left(\frac{1}{2}\right)^{k}=\sum_{k=1}^{\infty}\left(\frac{1}{2}\right)^{k+1} \\
& \quad=\frac{1 / 4}{1-1 / 2}=\frac{1}{2} .
\end{aligned}
$$

Therefore we have proven that

$$
\left|\left\|\ln \left(I+A_{n}\right)\right\|\left\|A_{n}\right\|^{-1}-1\right|<\frac{1}{2}, \quad \forall n>n_{0} ;
$$

that is,

$$
\frac{1}{2}<\frac{\left\|\ln \left(I+A_{n}\right)\right\|}{\left\|A_{n}\right\|}<\frac{3}{2}, \quad \forall n>n_{0} .
$$

On one hand, this leads to

$$
\left\|\ln \left(I+A_{n}\right)\right\|<\frac{3\left\|A_{n}\right\|}{2}, \quad \forall n>n_{0} .
$$

If we assume that $\sum_{n=1}^{\infty}\left\|A_{n}\right\|$ converges using a comparison test for positive numerical series we obtain that $\sum_{n=1}^{\infty} \| \ln (I+$ $\left.A_{n}\right) \|$ converges. This proves the converse implication in (19).

On the other hand, from (24) one gets

$$
\frac{\left\|A_{n}\right\|}{\left\|\ln \left(I+A_{n}\right)\right\|}<2, \quad \forall n>n_{0} ;
$$

that is,

$$
\left\|A_{n}\right\|<2\left\|\ln \left(I+A_{n}\right)\right\|, \quad \forall n>n_{0} .
$$

If we assume that $\sum_{n=1}^{\infty}\left\|\ln \left(I+A_{n}\right)\right\|$ converges, then again by a comparison test for positive numerical series we show that $\sum_{n=1}^{\infty}\left\|I+A_{n}\right\|$ converges, and the result is established.

Remark 14. Because of Theorems 10 and 13, it follows at once that an infinite matrix product which is absolutely convergent is also convergent since this property holds for matrix series.

Example 15. The infinite matrix product $\prod_{n=1}^{\infty}(I+(1 /(n+$ $1)(n+3)) I)$ is absolutely convergent. Notice that matrices $I+A_{n}$ with $A_{n}=(1 /(n+1)(n+3)) I$ satisfy that $A_{n} A_{m}=A_{m} A_{n}$ 
for every $n, m \geq 1$. In addition, none of the factors of the product is a singular matrix since

$$
-1 \notin \sigma\left(A_{n}\right)=\left\{\lambda_{n}: \lambda_{n}=\frac{1}{(n+1)(n+3)} \neq-1\right\},
$$

$\forall n \geq 1$.

Using Theorem 13 is enough to show that the matrix series $\sum_{n=1}^{\infty}(1 /(n+1)(n+3)) I$ is absolutely convergent. In fact,

$$
\begin{aligned}
\sum_{n=1}^{\infty}\left\|\frac{1}{(n+1)(n+3)} I\right\| & =\sum_{n=1}^{\infty} \frac{1}{(n+1)(n+3)}<\sum_{n=1}^{\infty} \frac{1}{n^{2}} \\
& <\infty .
\end{aligned}
$$

Moreover, it is easy to compute the value of the infinite product. For it, notice that

$$
\begin{aligned}
P_{n} & =\prod_{k=1}^{n}\left(I+\frac{1}{(k+1)(k+3)} I\right) \\
& =\prod_{k=1}^{n}\left(\frac{(k+1)(k+3)+1}{(k+1)(k+3)} I\right) \\
& =\prod_{k=1}^{n} \frac{(k+2)^{2}}{(k+1)(k+3)} I \\
& =\frac{[3 \cdot 4 \cdot 5 \cdots(n+2)]^{2}}{[2 \cdot 3 \cdot 4 \cdots(n+1)][4 \cdot 5 \cdot 6 \cdots(n+3)]} I \\
& =\frac{n+2}{2} \cdot \frac{3}{n+3} I .
\end{aligned}
$$

Then,

$$
\begin{aligned}
& \prod_{n=1}^{\infty}\left(I+\frac{1}{(n+1)(n+3)} I\right)=\lim _{n \rightarrow \infty} P_{n} \\
& \quad=\lim _{n \rightarrow \infty} \frac{3(n+2)}{2(n+3)} I=\frac{3}{2} I .
\end{aligned}
$$

Let us observe that the necessary condition of convergence for the infinite matrix product holds, since $\lim _{n \rightarrow \infty}(1 /(n+$ 1) $(n+3)) I=0 \in \mathbb{C}^{r \times r}$.

3.3. Uniform Convergence. In this section we introduce the concept of uniform convergence of an infinite matrix product, which plays a significant role in dealing with matrix functions defined by infinite products such as the matrix gamma function.

Definition 16. Let one assume that the factors of the matrix product depend on a complex variable $z$ and denote by $R$ a region of the complex plane $\mathbb{C}$. If this product converges in such a way that given any $\epsilon>0$ there exist $n_{0} \in \mathbb{N}$ independent of $z$, for all $z \in D$, such that

$$
\left\|\prod_{k=1}^{n_{0}+p}\left(I+A_{n}(z)\right)-\prod_{k=1}^{n_{0}}\left(I+A_{n}(z)\right)\right\|<\epsilon
$$

for all $p \in \mathbb{N}$, one says that the infinite product $\prod_{n=1}^{\infty}(I+$ $\left.A_{n}(z)\right)$ is uniformly convergent in the region $R$.

The following result provides a sufficient condition to guarantee uniform convergence of an infinite matrix product. Notice that it constitutes an analogous result like Weierstrass $M$-test for infinite matrix series.

Theorem 17. Let $R$ be a closed region of the complex plane and $\left\{A_{n}(z)\right\}_{n=1}^{\infty}$ a sequence of matrices in $\mathbb{C}^{r \times r}$ such that $-1 \notin$ $\sigma\left(A_{n}(z)\right), \forall n \geq 1$ and $z \in \mathbb{C}^{r \times r}$. Let one assume that there exists a sequence of positive numbers $\left\{m_{n}\right\}_{n=1}^{\infty}$ such that

$$
\begin{aligned}
& \left\|A_{n}(z)\right\|<m_{n}, \quad \forall n \geq 1, \forall z \in R, \\
& \sum_{n=1}^{\infty} m_{n}<\infty .
\end{aligned}
$$

Then $\prod_{n=1}^{\infty}\left(I+A_{n}(z)\right)$ is uniformly convergent in the region $R$.

Proof. By assumption $\sum_{n=1}^{\infty} m_{n}$ is convergent and $m_{n}>0$ for all $n \geq 1$; then using the scalar result analogous to Theorem 13 for infinite numerical products we conclude that $\prod_{n=1}^{\infty}\left(1+m_{n}\right)$ is convergent $[17, \mathrm{ch} .1]$. Therefore there exists $\lim _{n \rightarrow \infty} \prod_{k=1}^{n}\left(1+m_{k}\right)$. So, for any $\epsilon>0$, there is $n_{0} \in \mathbb{N}$, such that

$$
\prod_{k=1}^{n_{0}+p}\left(1+m_{k}\right)-\prod_{k=1}^{n_{0}}\left(1+m_{k}\right)<\epsilon
$$

for any natural number $p$.

By assumption, $\left\|A_{n}(z)\right\|<m_{n}, \forall n \geq 1$ and $\forall z \in R$. Thus by the submultiplicative property of the matrix norm one gets

$$
\begin{aligned}
& \left\|\prod_{k=1}^{n_{0}+p}\left(I+A_{k}(z)\right)-\prod_{k=1}^{n_{0}}\left(I+A_{k}(z)\right)\right\| \\
& \quad=\left\|\prod_{k=1}^{n_{0}}\left(I+A_{k}(z)\right)\left[\prod_{k=n_{0}+1}^{n_{0}+p}\left(I+A_{k}(z)\right)-I\right]\right\| \\
& \leq\left\|\prod_{k=1}^{n_{0}}\left(I+A_{k}(z)\right)\right\| \cdot\left\|\prod_{k=n_{0}+1}^{n_{0}+p}\left(I+A_{k}(z)\right)-I\right\| \\
& \quad \leq \prod_{k=1}^{n_{0}}\left(\left\|I+A_{k}(z)\right\|\right)\left[\prod_{k=n_{0}+1}^{n_{0}+p}\left(\left\|I+A_{k}(z)\right\|\right)-1\right]
\end{aligned}
$$

applying the triangle inequality and since $\|I\|=1$,

$$
\begin{aligned}
& \leq \prod_{k=1}^{n_{0}}\left(1+\left\|A_{k}(z)\right\|\right)\left[\prod_{k=n_{0}+1}^{n_{0}+p}\left(1+\left\|A_{k}(z)\right\|\right)-1\right] \\
& \leq \prod_{k=1}^{n_{0}}\left(1+m_{k}\right)\left[\prod_{k=n_{0}+1}^{n_{0}+p}\left(1+m_{k}\right)-1\right] \\
& =\prod_{k=1}^{n_{0}+p}\left(1+m_{k}\right)-\prod_{k=1}^{n_{0}}\left(1+m_{k}\right)<\epsilon
\end{aligned}
$$


where in the last step inequality (34) has been applied. This ends the proof.

\section{The Matrix Gamma Function}

In this section, we will apply the results presented in the foregoing section related to matrix infinite products in order to extend the definition of the Weierstrass gamma function to the matrix scenario.

Definition 18. Given $M \in \mathbb{C}^{r \times r}$ such that $-n \notin \sigma(M), \forall n \geq 1$, one defines the matrix gamma function, denoted by $\Gamma(M)$, as

$$
\Gamma^{-1}(M)=M e^{\gamma M} \prod_{n=1}^{\infty}\left[\left(I+\frac{1}{n} M\right) e^{-(1 / n) M}\right],
$$

where $\gamma$ is Euler-Mascheroni's constant defined by (2).

Let us show that this function is well defined by showing that such matrix infinite product is absolutely convergent. Rather than dealing with infinite matrix product (37), we will prove a more general statement; namely, we will prove that the matrix infinite product

$$
\prod_{n=1}^{\infty}\left(I-\frac{1}{c+n} A\right) e^{(1 / n) A}
$$

is absolutely convergent with $A \in \mathbb{C}^{r \times r}$ and $c \notin \mathbb{Z}-\mathbb{N}$; that is, $c$ is a nonpositive integer. Denoting $B_{n}=(I-(1 /(c+$ n))A) $e^{(1 / n) A}-I$, it is obvious that $B_{n} B_{m}=B_{m} B_{n}$ for every $n, m \geq 1$. By Theorem 13 it is sufficient to show that

$$
\sum_{n=1}^{\infty}\left[\left(I-\frac{1}{c+n} A\right) e^{(1 / n) A}-I\right]
$$

is absolutely convergent; that is, we have to show that the numerical series

$$
\sum_{n=1}^{\infty}\left\|\left[\left(I-\frac{1}{c+n} A\right) e^{(1 / n) A}-I\right]\right\|
$$

is convergent. For this, we will compare it with the convergent series $\sum_{n=1}^{\infty}\left(1 / n^{2}\right)$ and therefore it will be sufficient to show, by a comparison test, that

$$
\begin{gathered}
\lim _{n \rightarrow \infty} n^{2}\left\|\left(I-\frac{1}{c+n} A\right) e^{(1 / n) A}-I\right\|=a<\infty, \\
\lim _{n \rightarrow \infty} n^{2}\left\|\left(I-\frac{1}{c+n} A\right) e^{(1 / n) A}-I\right\| \\
=\left\|\lim _{n \rightarrow \infty} n^{2}\left[\left(I-\frac{1}{c+n} A\right) e^{(1 / n) A}-I\right]\right\|=
\end{gathered}
$$

to compute this limit, now we make the change of variable $\beta=1 /(c+n)$, so $n=(1-\beta c) / \beta$ and $1 / n=\beta /(1-\beta c)$; then

$$
\begin{aligned}
& =\left\|\lim _{\beta \rightarrow 0} \frac{(1-\beta c)^{2}}{\beta^{2}}\left[(I-\beta A) e^{(\beta /(1-\beta c)) A}-I\right]\right\| \\
& =\frac{1}{2}\|A(2 c I-A)\|=a<\infty,
\end{aligned}
$$

where the L'Hôpital rule has been applied twice in the last step. Therefore $\sum_{n=1}^{\infty}\left[(I-(1 /(c+n)) A) e^{(1 / n) A}-I\right]$ absolutely converges.

In particular, for $c=0$ we get that

$$
\prod_{n=1}^{\infty}\left(I-\frac{1}{n} A\right) e^{(1 / n) A}
$$

absolutely converges. Also, the result applies to $A=-M \epsilon$ $\mathbb{C}^{r \times r}$, so

$$
\prod_{n=1}^{\infty}\left(I+\frac{1}{n} M\right) e^{-(1 / n) M}
$$

absolutely converges and therefore

$$
\Gamma^{-1}(M)=M e^{\gamma M} \prod_{n=1}^{\infty}\left[\left(I+\frac{1}{n} M\right) e^{-(1 / n) M}\right]
$$

is absolutely convergent. Finally, notice that under the hypothesis $-n \notin \sigma(M), \forall n \geq 1$, none of the factors of the above infinite matrix product is singular since it is the product of two invertible matrices. Indeed, each of these factors is the product of the exponential matrix $e^{-(1 / n) M}$, which is invertible for all $n \geq 1$, and $B_{n}=I+(1 / n) M$. Since $\sigma\left(B_{n}\right)=\left\{\lambda_{n}: \lambda_{n}=\right.$ $1+(1 / n) \mu, \mu \in \sigma(M)\}$ and $\mu \neq-n$ for all $n \geq 1$, one gets $0 \notin \sigma\left(B_{n}\right) \forall n \geq 1$, which means that $B_{n}$ is invertible for each $n \geq 1$. Thus $\Gamma^{-1}(M)$ is well defined for each matrix $M \in \mathbb{C}^{r \times r}$ such that $-n \notin \sigma(M), \forall n \geq 1$. This extends to the matrix scenario the Weierstrass definition of the gamma function by an infinite product.

One of the most useful applications from our results is to prove the existence of certain matrix limits. Next, we present an illustrative example.

Example 19. Let us show that if $M \in \mathbb{C}^{r \times r}$ such that $-n \notin$ $\sigma(M)$ for all $n \geq 1$, then the following limit exists:

$$
\begin{aligned}
\lim _{n \rightarrow \infty}(n-1) ! \\
\cdot[(M+I)(M+2 I) \cdots(M+(n-1) I)]^{-1} n^{M},
\end{aligned}
$$

where $n^{M}=e^{M \ln n}$. As we will show later, this limit plays an important role in the study of the matrix gamma function. To verify that this limit exists, we consider an infinite product whose $n$th partial product $P_{n}$ coincides with the limit; that is,

$$
\begin{aligned}
P_{n} & =(n \\
& -1) ![(M+I)(M+2 I) \cdots(M+(n-1) I)]^{-1} n^{M} .
\end{aligned}
$$


If we prove that the infinite product converges, we conclude the existence of $\lim _{n \rightarrow \infty} P_{n}$. Let us observe that

$$
\begin{aligned}
P_{n+1} & =n ![(M+I)(M+2 I) \cdots(M+n I)]^{-1}(n+1)^{M} \\
= & n ![(M+n I) \cdots(M+2 I)(M+I)]^{-1}(n+1)^{M} \\
= & n !(M+I)^{-1} \cdot(M+2 I)^{-1} \cdots(M+n I)^{-1} \\
\cdot & (n+1)^{M}=\left[1(M+I)^{-1} 2^{M} 1^{-M}\right] \\
& \cdot\left[2(M+2 I)^{-1} 3^{M} 2^{-M}\right] \\
& \cdots\left[n(M+n I)^{-1}(n+1)^{M} n^{-M}\right] \\
= & \prod_{k=1}^{n}\left[k(M+k I)^{-1} \cdot(k+1)^{M} \cdot k^{-M}\right] \\
= & \prod_{k=1}^{n}\left[\left(\frac{1}{k} M+I\right)^{-1}\left(1+\frac{1}{k}\right)^{M}\right] .
\end{aligned}
$$

Consider the product $\prod_{n=1}^{\infty} B_{n}$ being $B_{n}=((1 / n) M+$ $I)^{-1}(1+1 / n)^{M}$. Since $B_{n} B_{m}=B_{m} B_{n}$ for every $n, m \geq 1$, to show that it absolutely converges, by Theorem 13 , it is enough to show that the matrix series

$$
\sum_{n=1}^{\infty}\left[\left(\frac{1}{n} M+I\right)^{-1}\left(1+\frac{1}{n}\right)^{M}-I\right]
$$

absolutely converges, that is, that the numerical series

$$
\sum_{n=1}^{\infty}\left\|\left(\frac{1}{n} M+I\right)^{-1}\left(1+\frac{1}{n}\right)^{M}-I\right\|
$$

converges. This is shown by comparison with the convergent series $\sum_{n=1}^{\infty}\left(1 / n^{2}\right)$. In fact, notice that

$$
\begin{aligned}
& \lim _{n \rightarrow \infty} n^{2}\left\|\left(\frac{1}{n} M+I\right)^{-1}\left(1+\frac{1}{n}\right)^{M}-I\right\| \\
& =\left\|\lim _{n \rightarrow \infty} n^{2}\left[\left(\frac{1}{n} M+I\right)^{-1}\left(1+\frac{1}{n}\right)^{M}-I\right]\right\|=
\end{aligned}
$$

and making the change of variable $\beta=1 / n$, one gets $n=1 / \beta$ and

$$
\begin{aligned}
& =\left\|\lim _{\beta \rightarrow 0} \frac{(M \beta+I)^{-1} \cdot(1+\beta)^{M}-I}{\beta^{2}}\right\| \\
& =\frac{1}{2}\|M(M-I)\|,
\end{aligned}
$$

where the L'Hôpital rule has been applied twice. Therefore, since the matrix infinite product

$$
\prod_{n=1}^{\infty}\left[\left(\frac{1}{n} M+I\right)^{-1}\left(1+\frac{1}{n}\right)^{M}\right]
$$

converges, then the matrix limit

$$
\begin{aligned}
& \lim _{n \rightarrow \infty} P_{n}=\lim _{n \rightarrow \infty}(n \\
& \quad-1) ![(M+I)(M+2 I) \cdots(M+(n-1) I)]^{-1} n^{M}
\end{aligned}
$$

exists. Notice that the assumption $-n \notin \sigma(M)$ for all $n \geq$ 1 guarantees that the product has no singular matrices as factors.

As a consequence we have the following limit representation of the matrix gamma function.

Theorem 20. If

$$
M \in \mathbb{C}^{r \times r}:-n \notin \sigma(M), \quad \forall n \geq 0,
$$

and $(M)_{n}$ denotes Pochhammer's matrix symbol $(M)_{n}=$ $M(M+I) \cdots(M+(n-1) I), n \geq 1,(M)_{0}=I$, then

$$
\Gamma(M)=\lim _{n \rightarrow \infty}(n-1) !\left((M)_{n}\right)^{-1} n^{N} .
$$

Proof. We know that

$$
\Gamma^{-1}(M)=M e^{\gamma M} \prod_{n=1}^{\infty}\left[\left(I+\frac{1}{n} M\right) e^{-(1 / n) M}\right]
$$

and by assumption $-n \notin \sigma(M)$, for all $n=1,2, \ldots, M+n I$ with $n \geq 1$ is invertible, and also the matrices $I+(1 / n) M$, for $n \geq 1$, are invertible since $I+(1 / n) M=(1 / n)(M+n I)$. With this fact and since the exponential matrix is invertible and that $\left(e^{M}\right)^{-1}=e^{-M}, \forall M \in \mathbb{C}^{r \times r}$, we get

$$
M \Gamma(M)=e^{-\gamma M} \prod_{n=1}^{\infty}\left[\left(I+\frac{1}{n} M\right)^{-1} e^{(1 / n) M}\right] .
$$

Then

$$
M \Gamma(M)=e^{-\gamma M} \lim _{n \rightarrow \infty} \prod_{k=1}^{n}\left[\left(I+\frac{1}{k} M\right)^{-1} e^{(1 / k) M}\right] .
$$

On the other hand, by (2) we know that

$$
\begin{aligned}
\gamma & =\lim _{n \rightarrow \infty}\left[H_{n}-\ln (n+1)\right] \\
& =\lim _{n \rightarrow \infty}\left[H_{n}-\sum_{k=1}^{n} \ln \left(\frac{k+1}{k}\right)\right], \quad H_{n}=\sum_{k=1}^{n} \frac{1}{k},
\end{aligned}
$$

and thus

$$
\begin{aligned}
e^{-\gamma M} & =e^{-\lim _{n \rightarrow \infty}\left[H_{n}-\sum_{k=1}^{n} \ln ((k+1) / k)\right] M} \\
& =\lim _{n \rightarrow \infty}\left\{e^{-H_{n} M+\left(\sum_{k=1}^{n} \ln ((k+1) / k)\right) M}\right\} \\
& =\lim _{n \rightarrow \infty}\left\{e^{-\left(\sum_{k=1}^{n}(1 / k)\right) M+\left(\sum_{k=1}^{n} \ln ((k+1) / k)\right) M}\right\} \\
& =\lim _{n \rightarrow \infty}\left\{e^{-\left(\sum_{k=1}^{n}(1 / k)\right) M} e^{\left(\sum_{k=1}^{n} \ln ((k+1) / k)\right) M}\right\} \\
& =\lim _{n \rightarrow \infty}\left\{\left[\prod_{k=1}^{n} e^{-(1 / k) M}\right]\left[\prod_{k=1}^{n}\left(\frac{k+1}{k}\right)^{M}\right]\right\} \\
& =\lim _{n \rightarrow \infty}\left\{\prod_{k=1}^{n}\left[e^{-(1 / k) M}\left(\frac{k+1}{k}\right)^{M}\right]\right\} .
\end{aligned}
$$


Substituting this last expression in (59),

$$
\begin{aligned}
& M \Gamma(M) \\
& =\lim _{n \rightarrow \infty}\left\{\prod_{k=1}^{n}\left[e^{-(1 / k) M}\left(\frac{k+1}{k}\right)^{M}\left(I+\frac{1}{k} M\right)^{-1} e^{(1 / k) M}\right]\right\} \\
& =\lim _{n \rightarrow \infty} \prod_{k=1}^{n}\left[\left(I+\frac{1}{k} M\right)^{-1}\left(\frac{k+1}{k}\right)^{M}\right] .
\end{aligned}
$$

It is already proven that the limit in the right-hand side exists and also that

$$
\begin{aligned}
& \lim _{n \rightarrow \infty} P_{n}=\lim _{n \rightarrow \infty}(n \\
& \quad-1) ![(M+I)(M+2 I) \cdots(M+(n-1) I)]^{-1} n^{M} ;
\end{aligned}
$$

therefore,

$$
\begin{aligned}
& M \Gamma(M)=\lim _{n \rightarrow \infty}(n \\
& \quad-1) ![(M+I)(M+2 I) \cdots(M+(n-1) I)]^{-1} n^{M},
\end{aligned}
$$

or taking into account that by hypothesis $0 \notin \sigma(M)$ this is equivalent to

$$
\begin{aligned}
& \Gamma(M)=\lim _{n \rightarrow \infty}(n \\
& -1) ![M(M+I)(M+2 I) \cdots(M+(n-1) I)]^{-1} n^{M} \\
& \quad=\lim _{n \rightarrow \infty}(n-1) !\left((M)_{n}\right)^{-1} n^{M} .
\end{aligned}
$$

\section{Conclusions}

In this paper we have introduced infinite matrix products and some of their main properties related to convergence. We have taken advantage of these results to extend the definition of the scalar gamma function by an infinite product to the matrix framework including a limit representation of this special function. The provided results can also be applied to generalize in the matrix sense numerous significant functions defined through infinite products. Apart from the scalar gamma function, specific examples of scalar functions defined by an infinite product are Weierstrass sigma function, Q-Pochhammer symbol, Ramanujan theta function, sinus function, Riemann zeta function, and so forth. Even more, according to the classical Weierstrass factorization theorem every entire function can be factored into an infinite product of entire functions. As a consequence, the obtained results about infinite matrix products are potentially applicable to a large class of functions beyond the matrix gamma function.

\section{Conflict of Interests}

The authors declare that there is no conflict of interests regarding the publication of this paper.

\section{Acknowledgment}

This work has been supported by Ministerio de Economía y Competitividad Grant MTM2013-41765-P.

\section{References}

[1] G. Kristensson, Second Order Differential Equations: Special Functions and Their Classification, Springer, New York, NY, USA, 2010.

[2] N. J. Vilenkin and A. U. Klimyk, Representation of Lie Groups and Special Functions, vol. 316 of Recent Advances Series: Mathematics and Its Applications, Springer, Berlin, Germany, 1995.

[3] J. E. Humphreys, Introduction to Lie Algebras and Representation Theory, vol. 9, Springer, New York, NY, USA, 1972.

[4] L. Jódar and E. Defez, "A connection between Laguerre's and Hermite's matrix polynomials," Applied Mathematics Letters, vol. 11, no. 1, pp. 13-17, 1998.

[5] K. A. M. Sayyed, M. S. Metwally, and M. T. Mohamed, "Certain hypergeometric matrix function," Scientiae Mathematicae Japonicae, vol. 69, no. 3, pp. 315-321, 2009.

[6] M. Abul-Dahab and A. Shehata, "A new extension of Humbert matrix function and their properties," Advances in Pure Mathematics, vol. 1, no. 6, pp. 315-321, 2011.

[7] A. Saboor, S. B. Provost, and M. Ahmad, "The moment generating function of a bivariate gamma-type distribution," Applied Mathematics and Computation, vol. 218, no. 24, pp. 11911-11921, 2012.

[8] J. Sastre and L. Jódar, "Asymptotics of the modified bessel and the incomplete gamma matrix functions," Applied Mathematics Letters, vol. 16, no. 6, pp. 815-820, 2003.

[9] A. Iranmanesh, M. Arashi, D. K. Nagar, and S. M. M. Tabatabaey, "On inverted matrix variate gamma distribution," Communications in Statistics-Theory and Methods, vol. 42, no. 1, pp. 28-41, 2013.

[10] J. Derbyshire, Prime Obsession: Bernhard Riemann and the Greatest Unsolved Problem in Mathematics, Penguin Books, New York, NY, USA, 2004.

[11] I. Daubechies and J. C. Lagarias, "Sets of matrices all infinite products of which converge," Linear Algebra and Its Applications, vol. 161, pp. 227-263, 1992.

[12] R. Bru, L. Elsner, and M. Neumann, "Convergence of infinite products of matrices and inner-outer iteration schemes," Electronic Transactions on Numerical Analysis, vol. 2, pp. 183-193, 1994.

[13] O. Holtz, "On convergence of infinite matrix products," Electronic Journal of Linear Algebra, vol. 7, pp. 178-181, 2000.

[14] R. A. Horn and C. R. Johnson, Matrix Analysis, Cambridge University Press, Cambridge, UK, 1985.

[15] G. H. Golub and C. F. van Loan, Matrix Computations, The Johns Hopkins University Press, Baltimore, Md, USA, 4th edition, 2013.

[16] W. J. Culver, "On the existence and uniqueness of the real logarithm of a matrix," Proceedings of the American Mathematical Society, vol. 17, no. 5, pp. 1146-1151, 1966.

[17] E. D. Rainville, Special Functions, Chelsea, New York, NY, USA, 1960. 


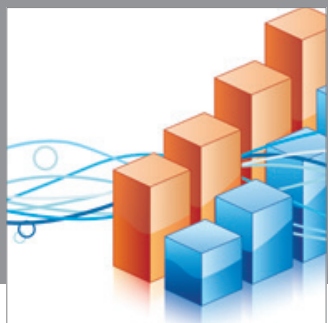

Advances in

Operations Research

mansans

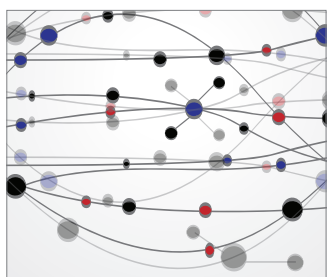

The Scientific World Journal
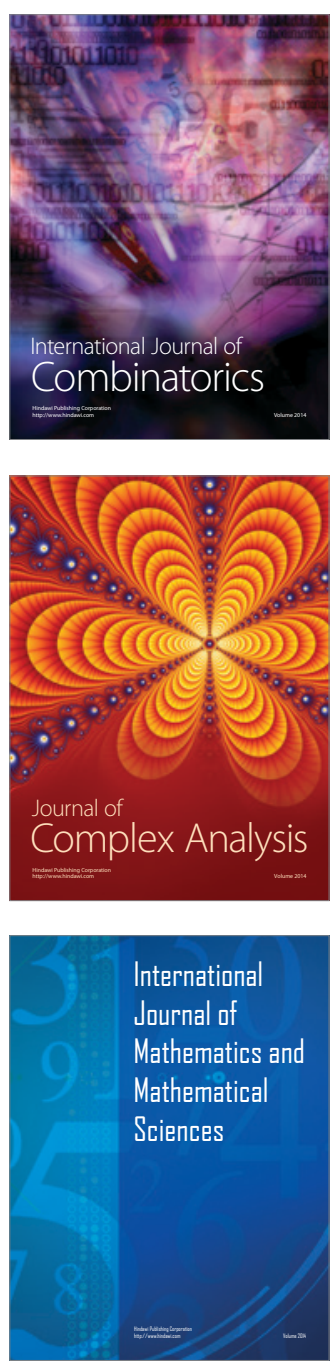
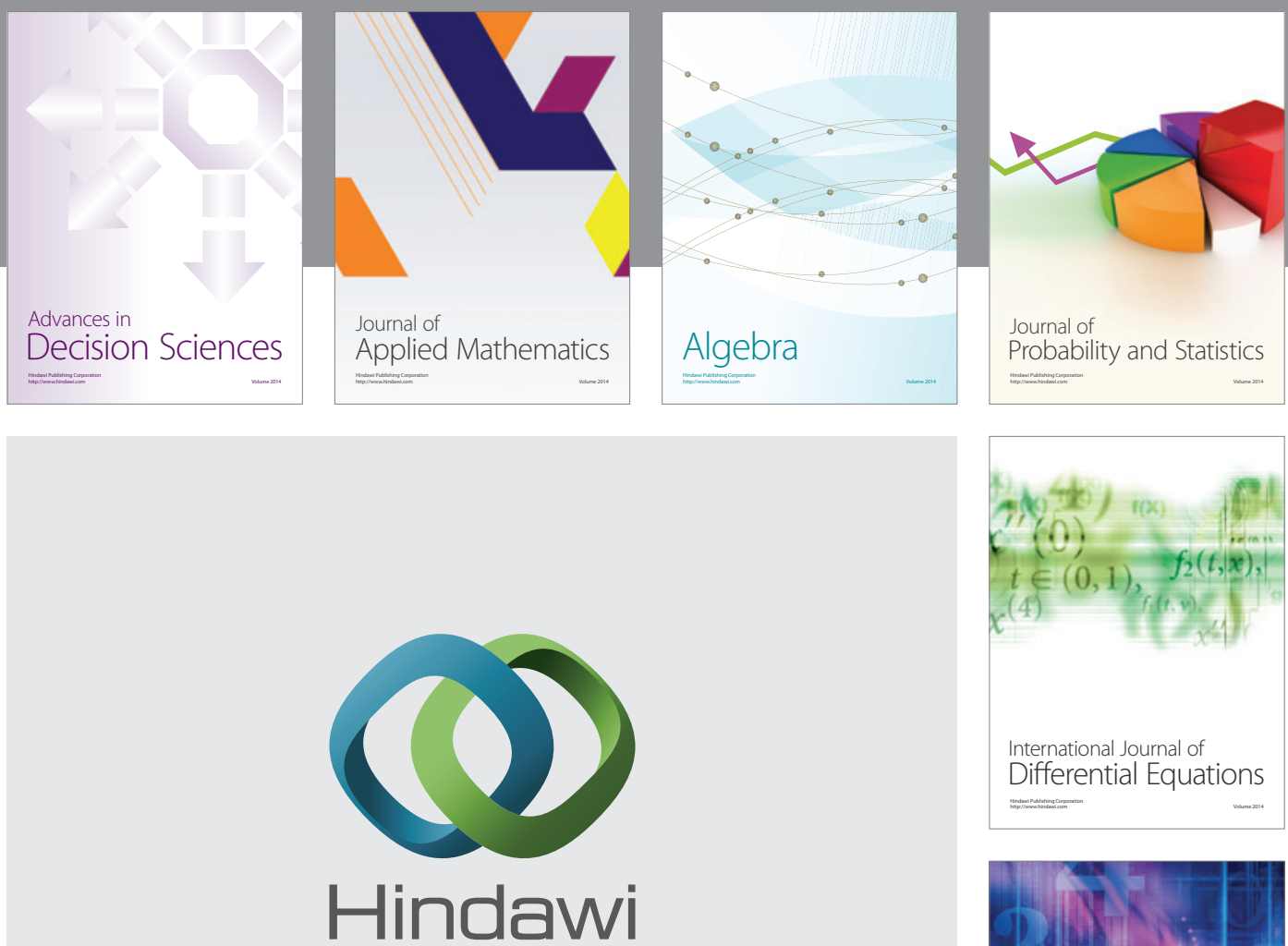

Submit your manuscripts at http://www.hindawi.com
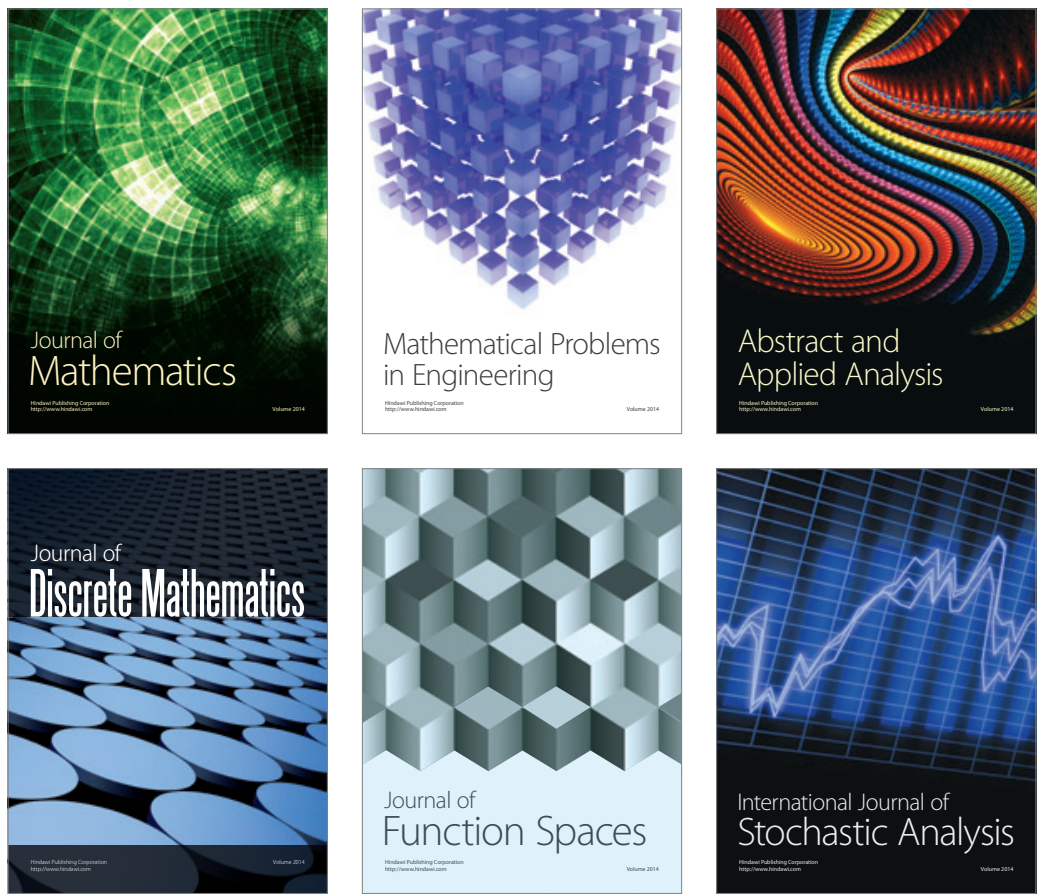

Journal of

Function Spaces

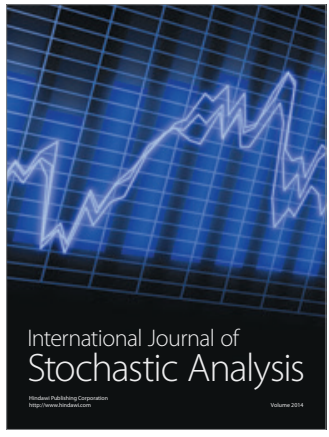

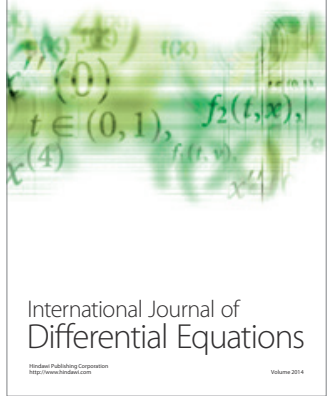
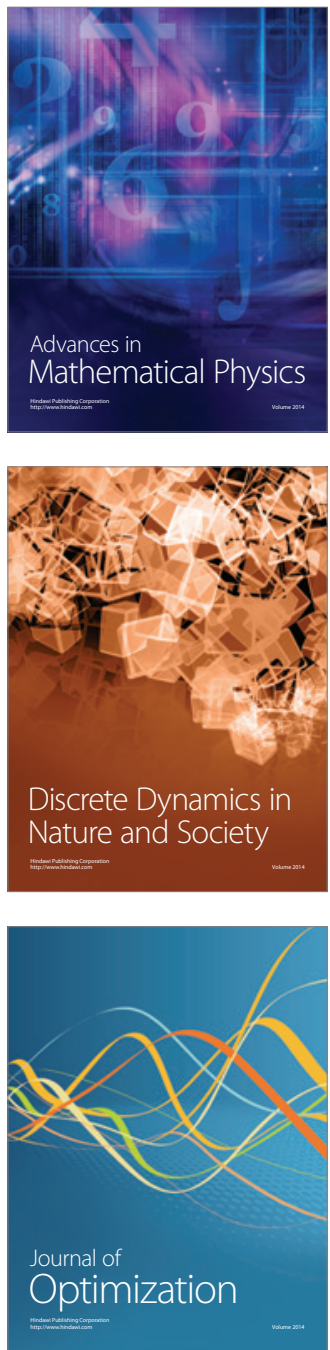